\title{
APPLICATION OF LASER-INDUCED BREAKDOWN CAVITATION BUBBLES FOR CELL LYSIS
} IN VITRO

\author{
DARINA JASIKOVA*, MIROSLAVA RYSOVA, MICHAL KOTEK \\ The Institute for Nanomaterials, Advanced Technology and Innovation, 1402/2, 461 17 Liberec 1, Czech Republic, Europe. \\ Email: darina.jasikova@tul.cz
}

Received: 10 November 2018, Revised and Accepted: 06 December 2018

\section{ABSTRACT}

Objective: Understanding the basic mechanism of the cavitation bubble action on living cells as a crucial step of development and application of sophisticated methods based on controlled cavitation in cell behaviour manipulation. Optimisation of parameters in order to expand cell lysis region created by a single bubble.

Methods: The cavitation bubbles are generated by the laser-induced breakdown method. The impact of controlled cavitation bubble on the biological system is synchronously monitored under a microscope and recorded. Visualization of the cavitation bubble course is monitored by a highspeed camera. The impact of technology on the healthy confluent cell layer is verified. Evaluation of the cavitation bubbles' effect on cells in real time and by subsequent analysis of the cell lysis region and impact of the cavitation bubble on cell viability is carried out by optical visualization and life/ dead fluorescence staining.

Results: Cavitation bubble induced in distance of $1.5 \mathrm{~mm}$ from the cell surface overcomes properties of sessile bubble and enables to create cell lysis region over $1000 \mu \mathrm{m}$ in diameter due to transient shear stress produced by liquid displaced by the bubble expansion.

Conclusion: Cell lysis region is strongly dependent on the spot laser energy (SLE) and the bubble induction distance from cells. This knowledge is crucial for application in chemical free cell lysis in vitro, wound induction for experimental purposes and cell layers patterning in desired scale.

Keywords: Cavitation bubble, Laser-induced breakdown, Cell lysis, Cell viability.

(C) 2019 The Authors. Published by Innovare Academic Sciences Pvt Ltd. This is an open access article under the CC BY license (http://creativecommons. org/licenses/by/4. 0/) DOI: http://dx.doi.org/10.22159/ijap.2019.v11s5.T3059

\section{INTRODUCTION}

The current trend in biotechnologies, including drug delivery, wound cleaning, and biofilm management is chemical-free and environmentally friendly processing based on cell lysis. There are various physical mechanisms, such as optical, mechanical, acoustical, and electrical, which can be applied as an alternative to chemical cell lysis and are capable of achieving the desired effect on cells. Among other techniques, laser based methods have been studied as relevant techniques for bacterial management with a potential in wound treatment applications. [1,2]. A comparison of the cell lysis method mentioned above is given in several review articles [3-6]. In specific cases, the cell lysis is mediated by cavitation bubbles induced individually or in a focused stream. One of the methods used to generate the cavitation bubble is laser-induced breakdown (LIB) [7-9]. This technique delivers energy with high spatial precision with respect to the biological sample. Among all of the cell lysis methods, laser-induced cavitation possess several advantages, such as its independence of cell type, its operation at lower temperatures, and its absence of chemical agents interacting problematically with some biochemical methods/compounds. Another indisputable advantage is its very precise definition of the beam, which is applicable for single cell lysis or is beneficial for cell lysis performed on a microfabrication platform [10]. LIB is based on a pulsed laser focused into microbeam. Basically, it involves thermal breakdown based on natural plasma generation. A plasma spot is exposed for a duration of microseconds to femtoseconds. This leads to direct, multiphoton, and cascade ionization. A significant role is played by impurities of the medium, spot size, light wavelength, and pulse width during the breakdown. The whole ionization mechanism is explained very well by Kennedy et al. [11]. The authors investigated the influence of the laser pulse duration and input laser energy on the bubble dynamics and the emission of shock waves. The energy distribution during the growth and collapse of a laser-induced bubble was described by Vogel et al. [12]. The effect of cavitation microbubbles on the cell substrate has already been described. The LIB generation mechanism through microscope lenses leads to the formation of cavitation bubbles of micrometer sizes. The advantage of these methods is the use of very low levels of energy $(\mu \mathrm{J})$ in femtosecond pulses to create a cavitation bubble in close proximity to the cell substrate, which is known as a sessile bubble. The defects induced by the cavitation microbubbles affect a very small area on the cell substrate, although the necrotic area of the cells can reach a diameter of up to $150 \mu \mathrm{m}[3]$.

The aim of this work was to explore the precise LIB generation of an individual enlarged cavitation bubble with a diameter of millimeters and evaluate its ability to sweep the surface area during its implosion defined by the process conditions. The distance and energy of the cavitation bubble were varied, and the effect on the cell lysis region and viability of cells present in the surrounding regions were evaluated. This application could be used in medical practice, for example, for highly precise chemical-free tissue lysis, sampling, and wound treatment.

\section{METHODS}

The basic element of the experimental arrangement was the Hellmas Analytics' cuvette, firmly established in space, being surrounded by the LIB device and a visualization setup. Cell monolayer samples were successively placed on the bottom of the cuvette filled with a physiological solution at a constant temperature. The experiment was designed for synchronous recording of the evolution and collapse of the cavitation bubble generated by the LIB device, while at the same time the impact zone in the cell layer was monitored using an inverted microscope. A diagram of the overall experimental setup is shown in Fig. 1. 
LIB device setup

The optical breakdown in the liquid is usually produced by focusing the laser light through suitably designed optics. In this work, we chose green light as an exciting light pulse. The absorption coefficient of the $532 \mathrm{~nm}$ laser light is only $0.02 \mathrm{~m}^{-1}$ in distilled water. This wavelength requires a higher energy input to the system to induce the cavitation process. The short, 10-ns laser pulse was generated using a Q-switched Nd: YAG New Wave Gemini pulse laser. The laser worked with one cavity for a single shot. The Q-switch signal synchronized the high-speed camera running in triggering mode, as shown in Fig. 1.

The LIB was set as an optical direct path. The outlet diameter of the laser beam was $5 \mathrm{~mm}$, with Gaussian characteristics of intensity. The magnification of the Galilean beam expander was calculated according to $\mathrm{MP}=\mathrm{EFL} 2 / \mid \mathrm{EFL} 1$. This setup was followed with the concave lens $\mathrm{f}_{\mathrm{s}}=300 \mathrm{~mm}$ and convex lens $\mathrm{f}_{\mathrm{s}}=75 \mathrm{~mm}$ with a diameter of $25 \mathrm{~mm}$. The focused laser beam created the laser point spot (radius $<0.1 \mathrm{~mm}$ ). Due to the losses in the optical path on each of the optical elements (reflections and refractions), comparable to this, we had to increase the energy level that enters the whole system. The set laser energy output is taken into account in relation to the bubble diameter, as shown in Fig. 2. The resulting cavitation bubble diameter was experimentally measured. The spot laser energy (SLE) was measured with an Ophir Vega laser power meter for slow pulse laser beams.

Another parameter necessary to be estimated before setting up the visualization technique is the cavitation bubble life span. This value is used to determine the scanning time as well as the sampling rate.

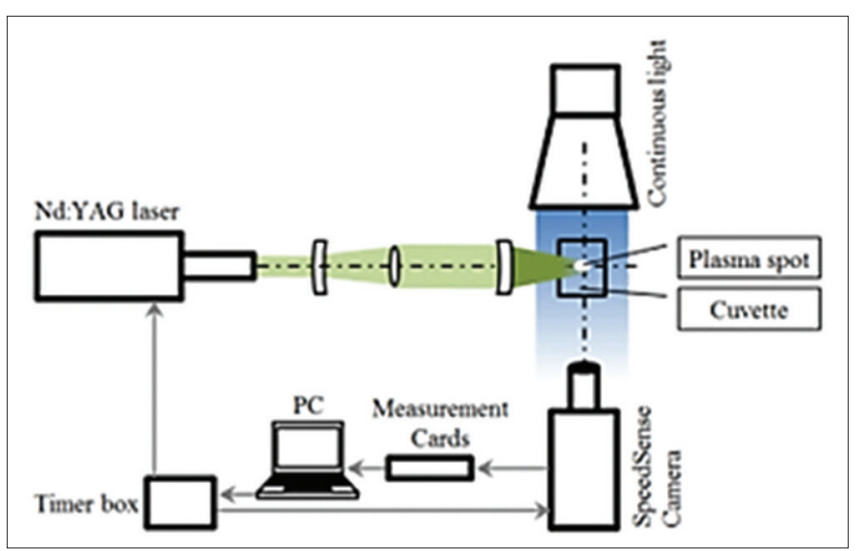

Fig. 1: The experimental setup showing the placement of the laser, cuvette, and the visualization system

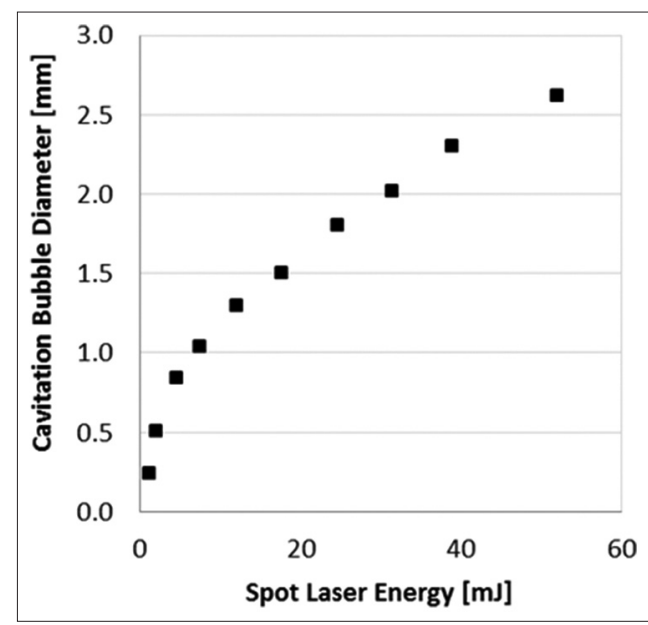

Fig. 2: Dependence of the cavitation bubble diameter on the inlet spot laser energy
The life span can be calculated using the following equation:

$t=0.915 \sqrt{\frac{\rho R^{2}}{p}}$

where $\rho$ is the density of the liquid, $\mathrm{R}$ is the radius of the cavitation bubble, and $\mathrm{p}$ is the difference between the atmospheric pressure and the vapor pressure within the expanded bubble $\left(2330 \mathrm{~Pa}\right.$ at $\left.20^{\circ} \mathrm{C}\right)$. For other experiments running in the cuvette, we chose laser input energies of $15 \mathrm{~mJ}, 30 \mathrm{~mJ}$, and $50 \mathrm{~mJ}$. This value corresponds to the cavitation bubble diameters of $1.34 \mathrm{~mm}, 2.20 \mathrm{~mm}$, and $2.63 \mathrm{~mm}$, respectively. The calculated and mathematically predicted life span of these cavitation bubbles is $139 \mu \mathrm{s}, 187 \mu \mathrm{s}$, and $243 \mu \mathrm{s}$, respectively. We were further able to adjust the sampling rate of the high-speed visualization, with knowledge of these complete times.

\section{Visualization setup}

The laser light came into the cuvette in the horizontal plane, and this direction was adapted to the speed camera visualization and scene lighting. We used a shadowgraphy setup for visualization of the cavitation bubble. This setup consisted of continuous LED matrix daylight lamp Veritas, MiniConstellation 120-5000 K of illuminance 92 Klux in $0.5 \mathrm{~m}$, equipped with an optical diffuse filter. A high-speed CMOS camera SpeedSense was placed opposite to the light source. This camera works at a frequency of $67.065 \mathrm{kHz}$ with a spatial resolution of $256 \times 256$ px or a lower frequency with a higher resolution of up to $1280 \times 800 \mathrm{px}$, and a 12 -bit dynamic range. The minimal camera exposure time was $1 \mu$ s. The camera was mounted with an optical lens system INFINIPROBE ${ }^{\text {TM }}$ TS-160 universal macro/micro-imaging system that enables $\times 4$ magnification. The lens was fitted with an edge pass and long pass filter cut-on wavelength $550 \mathrm{~nm}$ low pass optical filter to reduce the backward laser flashes to the camera and also to eliminate the flash generated during the plasmatic breakdown. The camera was operated in the triggering mode. The trigger was activated with the Q-switch pulse of laser for LIB. Once the motion was captured, its dataset was transferred to a computer. The starting point of the synchronization was the plasma spot accompanied by the flash.

The visualization system was completed with a microscope. The visualization system for cell observation was built on a Leica inverted microscope. The microscope was fitted with a Basler acA2040-180kc industrial camera running in continuous mode. The purpose of the camera was to monitor the immediate state of the cell layer immediately after the cavitation.

\section{Cell seeding and culture}

The study was performed on adherent $3 \mathrm{~T} 3$ clone A31 murine fibroblasts. The cell line was chosen based on the skin as a tissue of interest and due to its altered metabolism in comparison to normal human fibroblast. The cells were cultured in complete culture media consisting of high glucose Dulbecco's Modified Eagle Medium (DMEM, Sigma-Aldrich), 5\% fetal bovine serum (Tico), $5 \%$ newborn calf serum, and $1 \%$ penicillinstreptomycin antibiotic solution (Pen/Strep, $10 \mathrm{kU}$ penicillin and $10 \mathrm{mg}$ streptomycin per $\mathrm{mL}$, Sigma-Aldrich). During the pre-culture period, the cells were passaged every 3-4 days and kept in a log phase.

Before cell seeding, $24 \mathrm{~mm}$ round coverslips were pre-treated to increase cell and secure homogenous cell spreading. The coverslips were washed and placed into a $1 \mathrm{M} \mathrm{NaOH}$ bath overnight. The base bath was followed by a repeated bath in ultrapure water for $60 \mathrm{~min}$ each at room temperature, $95 \%$ ethanol bath, and bath again. The treated coverslips were sterilized by dry heat $\left(140^{\circ} \mathrm{C} / 60 \mathrm{~min}\right)$ and stored until used.

On the day of cell seeding, the coverslips were placed into a 6-well nontreated culture plate and equilibrated in complete culture media for $1 \mathrm{~h}$ $\left(37^{\circ} \mathrm{C}\right)$. 3T3_A31 cells (passage n. 21) were seeded on the coverslips at a density of 25.000 cells $/ \mathrm{cm}^{2}$ and cultured in a complete medium 
supplemented with phenol red as an indication until full confluence. The coverslips were seeded in duplicate for each experiment.

\section{Cell viability in the wound area}

Cell viability was evaluated in regions surrounding the wound site (impact zone). $4 \mathrm{~h}$ after the wound induction, cell-seeded coverslips were washed in Dulbecco's phosphate-buffered saline (D-PBS) w/o calcium and magnesium (D-PBS, Fisher Scientific) twice and living/dead fluorescent staining was performed. Briefly, solutions of ethidium homodimer-1 (EthD-1) (Fisher Scientific) and calcein acetoxymethyl (calcein AM) ( $\geq 96 \%$, Sigma-Aldrich) were prepared in no phenol red containing a high glucose DMEM medium (SigmaAldrich) in concentrations of $5 \mathrm{mM}$. The washed coverslips were incubated in a staining solution for $1 \mathrm{~h}$ under standard conditions $\left(37^{\circ} \mathrm{C}\right.$ and $\left.5 \% \mathrm{CO}_{2}\right)$ and washed 3 times in D-PBS. After the staining, the coverslips were mounted without fixation and observed using a fluorescence microscope. The imaging was carried out on an Axio Imager M2 microscope using EC Plan-Neofluar objectives $2.5 \times-40 \times$ (Carl Zeiss). This method was used to locate and distinguish dead cells in the cavitation bubble impact area as the EThD-1 is able to penetrate damaged cell membranes and turn fluorescent when bound to nuclei acids, whereas calcein AM is converted into its fluorescent form by intracellular esterases only in living cells. The fluorescence of these dyes is characteristic by $\lambda$ ex $\sim 495 \mathrm{~nm} / \lambda \mathrm{em} \sim 635 \mathrm{~nm}$ for EthD- 1 and $\lambda$ ex $\sim 495 \mathrm{~nm} / \lambda \mathrm{em} \sim 515 \mathrm{~nm}$ for calcein. The distribution and ratio of the living and dead cells were evaluated according to their distance from the wound edge.

\section{RESULTS AND DISCUSSION}

Cavitation bubbles were generated at three distances from the sample surface: $0 \mathrm{~mm}$ (sessile bubble), $1.5 \mathrm{~mm}$, and $3 \mathrm{~mm}$. Three sets of SLE input energies were set for each distance: $15 \mathrm{~mJ}, 30 \mathrm{~mJ}$, and $50 \mathrm{~mJ}$. The energy of the laser pulse was measured just in the focused spot, which further generated the plasma in the liquid. The cavitation bubble is characterized by its formation, expansion, and subsequent collapse. The greatest effect on the cell layer can be expected during the collapse when the accelerated jet of overheated vapors moves toward the surface. The duration of each phase is dependent on the initial SLE input laser energy, Table 1. The collapse of the cavitation bubble is followed by the jet formation. The jet velocity is higher than the velocity of the bubble surface. The maximal jet velocities are approximately $200 \mathrm{~m} . \mathrm{s}^{-1}$, and their diameter is assumed to be $1 / 60$ of the maximum bubble diameter [12]. A shock wave is present that affects the viability of cells over a larger area.

We hereby present the development of the cavitation bubbles at different time phases and SLEs. The importance of the jet impact characteristics was confirmed as the surface area impacted by the jet was strongly dependent on the bubble diameter and the distance from the sample surface. A representative time-lapse of the bubble development and duration of individual phases is presented in Table 1 and Fig. 3.

A comparison of the cavitation bubbles generated on the surface (sessile bubble) and in the distance is shown in Fig. 4. The images show the intensity of the cavitation bubble energy impact during the collapse. The generation of sessile cavitation bubbles is preferred by most authors because a greater zone of impact is expected as the collapse of the bubble is fully projected onto the surface.

The aim of our research was to find a position - distance of the cavitation bubble above the surface that causes extended damage to the cell layer. No demonstrable effects were determined at a distance of $3 \mathrm{~mm}$ from the surface. This distance corresponds to approximately $\times 3$ the radius of the maximum bubble size. The effect of an accelerated jet was not proven. The energy developed during the implosion and collapse is fully spent by the liquid in the vicinity. Here, we assume that closer to the surface; the energy is more focused on it. The rate of emitted energy into the vicinity is decreased. This is the main reason, why we assume it is more convenient to place the cavitation bubbles above the surface rather than a sessile bubble.
Table 1: Duration of expansion, collapse, and the impacting phase of the cavitation bubbles. The table states the mean values of 10 measured cavitation processes

\begin{tabular}{llll}
\hline $\begin{array}{l}\text { Spot laser }(\mathrm{mJ}) \\
\text { energy }\end{array}$ & $\begin{array}{l}\text { Expansion } \\
\text { phase }(\boldsymbol{\mu} \mathbf{s})\end{array}$ & $\begin{array}{l}\text { Collapse } \\
\text { phase }(\boldsymbol{\mu} \mathbf{s})\end{array}$ & $\begin{array}{l}\text { Impacting/ } \\
\text { jet phase }(\boldsymbol{\mu s})\end{array}$ \\
\hline 15 & 45 & 60 & 150 \\
30 & 90 & 90 & 135 \\
50 & 105 & 120 & 150 \\
\hline
\end{tabular}

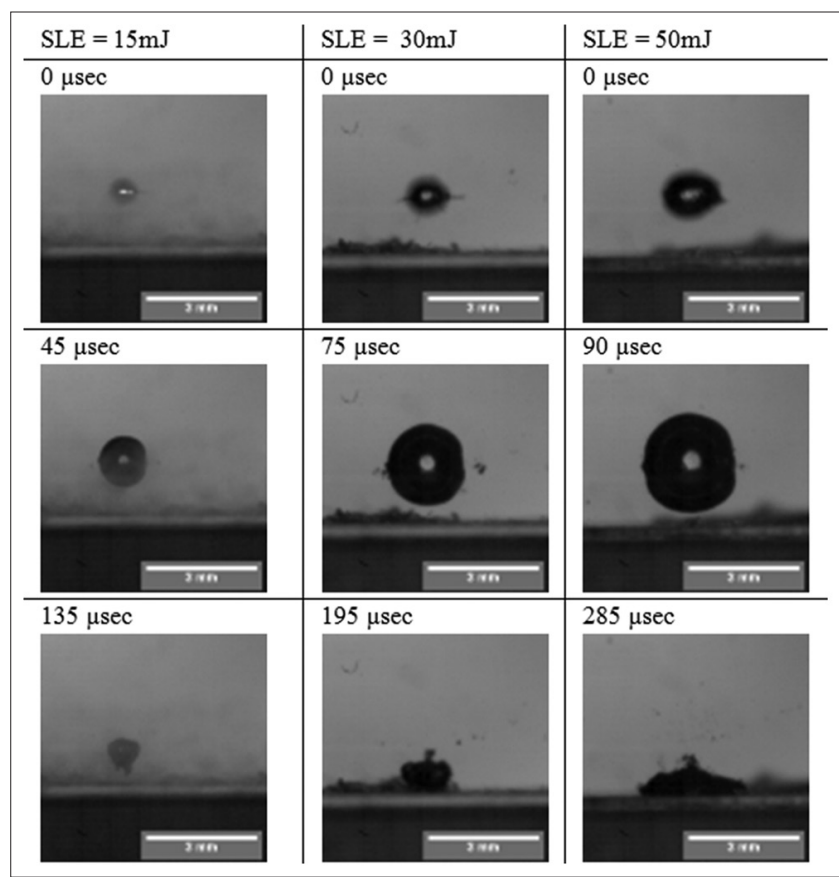

Fig. 3: Development of the cavitation bubbles over time. The effect of the energy input on the size of the bubble-induced $1.5 \mathrm{~mm}$ from the cell layer surface. Scale bar $=3 \mathrm{~mm}$

The most effective impact of collapsing the cavitation bubble was achieved at a distance of $1.5 \mathrm{~mm}$, which corresponds to $\times 2$ the radius of the maximum bubble size. A pressure wave is formed around the cavitation bubble at this distance. It is one of the mechanisms that have a major effect on the defect on the cell layers. The initial cell layer defect corresponds to the bubble diameter or is slightly larger due to the associated effect of the pressure wave action. This effect is assumed to be related to transient shear stress produced by liquid displaced by the expansion of the cavitation bubble $[13,14]$. The mean diameter of the cell lysis region $\left(\mathrm{R}_{\mathrm{LYS}}\right)$ obtained after the bubble impact was $1434 \mu \mathrm{m}$ in the case of the $1.5 \mathrm{~mm} / 50 \mathrm{SLE}$, which is almost double the size compared to the $884 \mu \mathrm{m}$ cell lysis region obtained by the $0 \mathrm{~mm} / 650$ SLE sessile bubble.

The living/dead staining expanded the obtained information on the cavitation bubble - cell culture interactions and revealed the impact of the process parameters on the cell apoptosis around the lysis region. In contrary to the results of Hellman et al. [3], who during their experiment produced targeted single cells and cell lysis regions of up to $300 \mu \mathrm{m}$ surrounded by viable cells, we found that the distance modification combined with optimal SLE leads to the formation of a cavitation bubble with a much wider and more complex impact zone. It consists of an $\mathrm{R}_{\mathrm{LYS}}$ region, where cells are completely eliminated from the glass surface, and an $\mathrm{R}_{\mathrm{NECR}}$ region, which is an area forming an edge on the $\mathrm{R}_{\mathrm{LYS}}$ region and mainly contains necrotic cells. This edge is surrounded by a region of necrotic/surviving cells $\left(\mathrm{R}_{\text {SURV }}\right)$, whose ratio changes depending on the distance from the center of the bubble impact zone. 


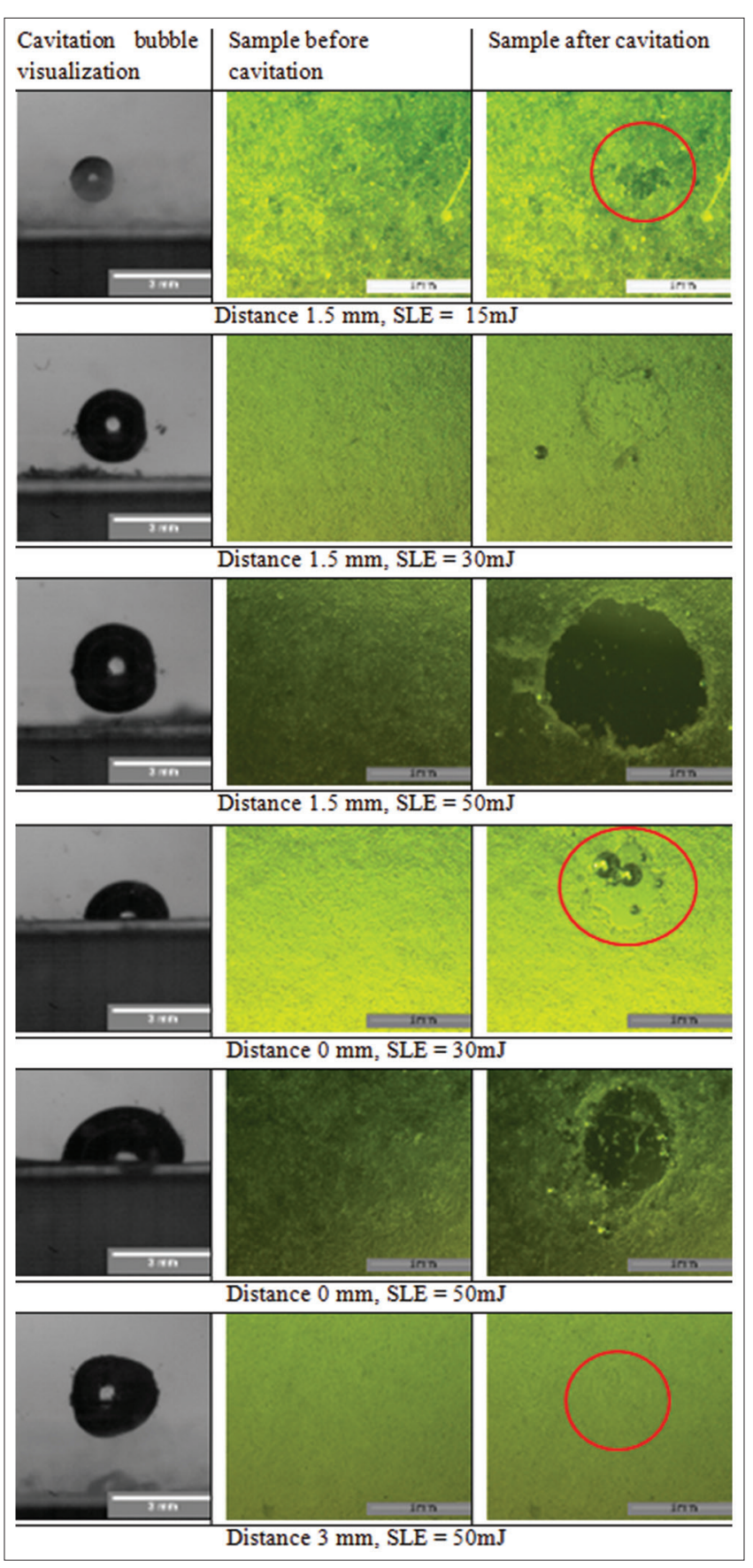

Fig. 4: Impact of the bubble on the cell layer. Comparison of bubble size and wound (cell lysis region) induced by its impact. Scale bars: white $=3 \mathrm{~mm}$, gray $=1 \mathrm{~mm}$

The size and characteristics of the $\mathrm{R}_{\text {SURV }}$ region strongly depend on the cavitation bubble. This region is surrounded by viable cells. We observed the organization of the impact zone in this way in all of the samples except for the cavitation bubbles formed at a distance of $3 \mathrm{~mm}$. In the case of these bubbles, no cell lysis was observed. The application of 50 $\mathrm{mJ}$ SLE led to the formation of a small and well-defined necrotic region directly surrounded by viable cells (Fig. 5 a). The regions surrounding the lysis region produced by the sessile bubble $(0 \mathrm{~mm} / 50 \mathrm{~mJ})$ are presented in Fig. $5 b$. The EthD-1 staining reveals the well-defined $\mathrm{R}_{\mathrm{NECR}}$ region surrounded by the $R_{\text {SURv }}$ region. The proportion of viable and necrotic cells in the $\mathrm{R}_{\text {SURV }}$ region is distinguished by the colors in Fig. $5 \mathrm{c}$, where green spots show the viable cells and red spots the necrotic ones. The distance from the wound edge showing the direction of the pressure wave formed after the bubble impact is marked.

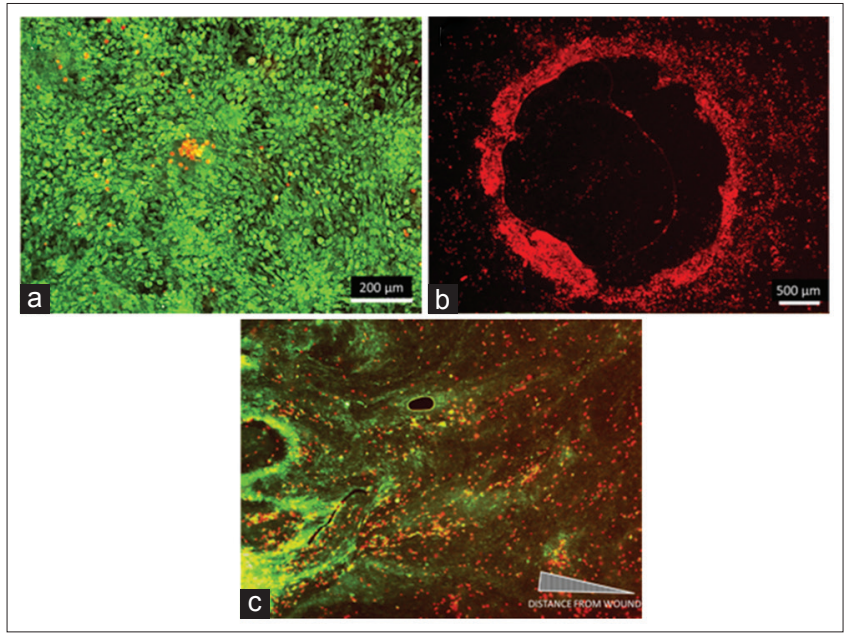

Fig. 5: Fluorescence microscopy results showing the impact of the cavitation bubble on the fibroblast cell culture by L/D staining (green - living cells, red-dead cells). (a) Impact zone generated by a $3 \mathrm{~mm} / 50 \mathrm{~mJ}$ bubble (mag. ×100), (b) impact zone generated by a $1.5 \mathrm{~mm} / 50 \mathrm{~mJ}$ bubble with only necrotic cells visualized (mag. $\times 25$ ), (c) L/D staining showing cell distribution in the $\mathbf{R}_{\text {SURV }}$ region of $1.5 \mathrm{~mm} / 50 \mathrm{~mJ}$ (mag. $\times 40)$

\section{CONCLUSIONS}

This paper defined the methodology of the cavitation bubble process involving bubble generation and its visualization. Cavitation was used for cell lysis and to determine the most suitable and effective geometrical and input energy parameters of the system. To achieve this, we evaluated the setup based on the position of the cavitation bubble above the cell layer at a distance of $\times 2$ the radius of the maximum size of the bubble. The viability of the cells depending on the properties of the cavitation bubble formation and distance from the impacted cell layer were studied. According to our results, the previously described $\mathrm{R}_{\mathrm{NECR}}$ region using our experimental setup is surrounded by an $\mathrm{R}_{\text {SURV }}$ region, which consists of a combination of viable and necrotic cells. The cell viability increased with distance from the center of the impact zone wound edge. The characteristics of this region are strongly dependent on the size and distance of the cavitation bubble.

\section{ACKNOWLEDGMENTS}

This work was supported by Institutional Research Program 2018 at the Faculty of Mechatronics, Informatics and Interdisciplinary Studies at the Technical University of Liberec and by the Ministry of Education, Youth and Sports of the Czech Republic and the European Union -European Structural and Investment Funds in the frames of Operational Programme Research, Development and Education project Hybrid Materials for Hierarchical Structures (HyHi, Reg. No. CZ.02.1.01/0.0/0.0/16_019/0000843).

\section{REFERENCES}

1. Sandini A, Meidyawati RK, Npa DA. The antibacterial affect of a diode laser used as an adjunct irrigant on clinical isolate of Enterococcus Faecalis biofilm in vitro. Int J App Pharm 2018;9:103-6.

2. Iselinni C, Meidyawati R, Djauharie N. Effects of a 980- nm diode laser's activation of $2.5 \% \mathrm{NaOCl}$ and $2 \%$ chlorhexidine antifungal irrigation solutions on Candida albicans biofilms. Int J App Pharm 2018;9:14-6.

3. Hellman AN, Rau KR, Yoon HH, Venugopalan V. Biophysical response to pulsed laser microbeam-induced cell lysis and molecular delivery. J Biophotonics 2008;1:24-35.

4. Rau KR, Guerra A. Investigation of laser-induced cell lysis using rime resolved imaging. Appl Phy Lett 2004;84:2940-2.

5. Rau KR, Quinto-Su PA, Hellman AN, Venugopalan V. Pulsed laser microbeam-induced cell lysis: Time-resolved imaging and analysis of hydrodynamic effects. Biophys J 2006;91:317-29. 
6. Brown RB, Audet J. Current techniques for single-cell lysis. J R Soc Interface 2008;5 Suppl 2:S131-8.

7. Brennen CE. Cavitation and Bubble Dynamics. New York: Cambridge University Press; 2015. p. 59-88.

8. Brujan EA, Nahen K, Schmidt P, Vogel A. Dynamics of laser-induced cavitation bubbles near elastic boundary. J Fluid Mech 2001;433:25181.

9. Jasikova D, Muller M, Kotek M, Kopecky V. The synchronized force impact measurement and visualization of single cavitation bubblegenerated with LIB. Int J Mech 2015;9:76-82.

10. Islam MS. A review on macroscale and microscale cell lysis methods.
Micromachines 2017;8:83

11. Kennedy PK, Hammer DX, Rockwell BA. Laser-induced breakdown in aqueous media. Prog Quantum Electron 1997;21:155-248.

12. Vogel A, Noack J, Nahen K, Theisen D, Busch S, Parlitz U. Energy balance of optical breakdown in water at nanosecond to femtosecond time scales. Appl Phys B 1999;68:271-80.

13. Brujan EA, Nahen K, Schmidt P, Vogel A. Dynamics of laser-induced cavitation bubbles near an elastic boundary. J Fluid Mech 2001;133:251-81.

14. Compton JL, Hellman AN, Venugopalan V. Hydrodynamic determinants of cell necrosis and molecular delivery produced by pulsed laser microbeam irradiation of adherent cells. Biophys J 2013;105:2221-31. 